(2) Open Access Full Text Article

REVIEW

\title{
Efficacy and safety of modified-release prednisone in patients with rheumatoid arthritis
}

This article was published in the following Dove Press journal:

Drug Design, Development and Therapy

8 March 2016

Number of times this article has been viewed

\section{Marco Krasselt \\ Christoph Baerwald}

Rheumatology Unit, Clinic for Gastroenterology and Rheumatology, Department of Internal Medicine, Neurology and Dermatology, University of Leipzig, Leipzig, Germany
Correspondence: Marco Krasselt Rheumatology Unit, Clinic for Gastroenterology and Rheumatology, Department of Internal Medicine, Neurology and Dermatology, University of Leipzig, Liebigstr 20, 04103 Leipzig, Germany

Tel +493419724710

Fax +49 34I 9724709

Email marco.krasselt@medizin.uni-leipzig. de

\begin{abstract}
The introduction of modified-release (MR) prednisone adds a drug with encouraging potential to the armamentarium of the rheumatologist. In particular, for patients experiencing a reduced quality of life due to prolonged morning stiffness, it is a promising therapeutic approach. Two clinical trials and one open-label observational study investigated the effectiveness of MR prednisone in reducing rheumatoid arthritis-related morning stiffness for both new and current users of corticosteroids. The efficacy and safety of MR prednisone use in rheumatoid arthritis patients are reviewed in this article. This includes pivotal trials as well as pathophysiological considerations and clinical implications.
\end{abstract}

Keywords: modified-release prednisone, rheumatoid arthritis, efficacy, chronotherapy, HPA axis, side effects, low-dose prednisone

\section{Introduction}

Rheumatoid arthritis

Epidemiology

Rheumatoid arthritis (RA) is a common autoinflammatory disease with an estimated incidence of approximately 41/100,000 per year. Incidence rates are rising with age up to $89 / 100,000$ people at the age of $70 .{ }^{1}$ With a lifetime risk of $3.6 \%$, women are affected considerably more frequently than men (risk: $1.7 \%$ ). ${ }^{2}$ The overall prevalence in the general population is shown to be almost $1 \%{ }^{3} \mathrm{RA}$ is the most frequent cause of an inflammatory arthritis.

\section{Clinical presentation}

Patients with RA typically suffer from symmetrical joint swelling, morning stiffness, and pain, caused by an inflammatory synovitis. RA usually involves the small joints of the fingers and toes, preferentially inflaming the proximal interphalangeal and metacarpophalangeal joints, but also wrists, ankles, and knees. The synovitis is often accompanied by unspecific constitutional symptoms, such as fatigue, fever, sweats, and weight loss. Untreated, it potentially leads to a disabling joint destruction with loss of function. Rapid therapy induction at an early disease stage not only reduces the risk of such a loss of function but also increases remission rates. ${ }^{4}$

In particular, after a long-standing disease, a variety of extra-articular manifestations, such as heart involvement, vasculitis, or interstitial lung disease, can occur. Among these comorbidities, diseases of the heart are particularly pronounced, possibly due to chronic systemic inflammation. Congestive heart failure and ischemic heart disease are not only more common in RA patients than in the general population but are also associated with an increased mortality. ${ }^{5}$ 


\section{Morning function}

In everyday life, the impairing joint stiffness, particularly in the morning hours, is one of the biggest clinical problems besides the joint pain. It is frequently reported in RA patients ${ }^{6}$ and negatively affects the individual quality of life. ${ }^{7}$ While patients with osteoarthritis also sometimes suffer from stiffness, it is much more common in RA. Different to osteoarthritis, it lasts longer, particularly after resting the joints, and is associated with disease activity.

Besides swollen or tender joints and laboratory parameters, rheumatologists used the morning stiffness for disease classification. The American College of Rheumatology (ACR) classification criteria for RA from 1987 listed morning stiffness with a duration of at least 1 hour as one criterion for classifying an arthritis as RA. ${ }^{8}$ The 2010 revised criteria though did no longer include morning stiffness as a classification criterion; ${ }^{9}$ this was probably due to the fact that other forms of inflammatory arthritis also go along with morning stiffness. The updated criteria reach a higher diagnostic specificity (96\%) compared to the old version $(86 \%) .{ }^{10}$

Taking together the higher specificity of these new criteria as well as the clinically established use of anticitrullinated proteins/peptides antibodies, definite RA can be detected earlier. These improvements are accompanied by the early induction of an anti-inflammatory therapy with a greater variety of potent drugs. It is important to mention the tumor necrosis factor (TNF) inhibitors here, the development of which was a huge step forward in treating RA patients successfully.

While the "severity" of morning stiffness is strongly correlated with morning joint pain and at least moderately with disease activity (Disease Activity Score 28 joints [DAS28]) and DAS28 response, morning stiffness "duration" only shows a weak correlation to measures of disease activity. ${ }^{11}$ The mere length of morning stiffness is therefore not suitable for the rheumatologist to conclude on disease activity. In concordance, patients hypothetically would pay more for a reduction in morning stiffness severity than a reduction in stiffness duration. ${ }^{12}$

However, it is important to mention that joint stiffness is a complex symptom. For reasons of simplification, we usually describe it in terms of duration and severity, but for the RA patient himself or herself, its impact on physical function or the ability to work is much more important in everyday life. ${ }^{13}$

\section{The role of chronobiology in morning stiffness}

The pathophysiology of RA is mainly driven by inflammatory cytokines, such as TNF and interleukin 6 (IL-6), which are preferentially produced by pathological monocyte subpopulations. ${ }^{14-16}$ The pivotal role of these cytokines is emphasized by the clinical efficacy of specific cytokine inhibitors, such as etanercept or tocilizumab. Distinct cytokine patterns of monocytes deriving from RA patients might even be able to predict therapy response in advance. ${ }^{17,18}$

Production and secretion of inflammatory cytokines show a dependence on the human body's day-night cycle resulting in changing cytokine plasma levels over 24 hours. A substantial variability during the night in RA patients was shown for IL- $6 .{ }^{19}$ Consequently, the state of inflammation and therefore the clinical symptoms vary in dependence of the daytime..$^{19,20}$

Most important in the context of RA is the observation that joint stiffness usually occurs in the morning hours. This can be readily explained by high cytokine levels in the morning hours ${ }^{19}$ as well as edema of the synovium and periarticular structures with redistribution of interstitial fluid while sleeping. ${ }^{21}$

The production of the typical cytokines, in particular IL-6, usually rises around 3 am in the morning. At the same time, endogenous corticosteroids are produced at a low level only and do not reach their peak levels before 7-8 am (Figure 1). ${ }^{22}$ Both the circadian rhythm of cytokine secretion on one hand and insufficient production of endogenous cortisol on the other hand are probably the most important reasons for the described symptoms being worse in the morning. These findings determine the optimal time for administration of exogenous glucocorticoids (GCs) as illustrated in Figure 1.

Eventually, this hypothetic rationale was clinically proven first by Arvidson et al; ${ }^{23}$ in a small trial, they showed that application of low-dose prednisone at 2 am leads to a more pronounced and significant reduction of joint stiffness, pain, and morning serum IL-6 levels in comparison to an administration at $7.30 \mathrm{am}$.

\section{The HPA axis}

IL-6 not only rises in the early morning hours, it also seems to stimulate the hypothalamic-pituitary-adrenal (HPA) axis resulting in increased cortisol plasma levels. However, chronic inflammation with repeatedly elevated IL-6 levels seems to lead to an adaption of the HPA response. ${ }^{24}$ The endogenous cortisol secretion in response to IL- 6 becomes inadequate in relation to the ongoing inflammation; this concept, being called "HPA axis insufficiency", was shown in RA patient ${ }^{25}$ and properly explains the dilemma shown in Figure 1. 


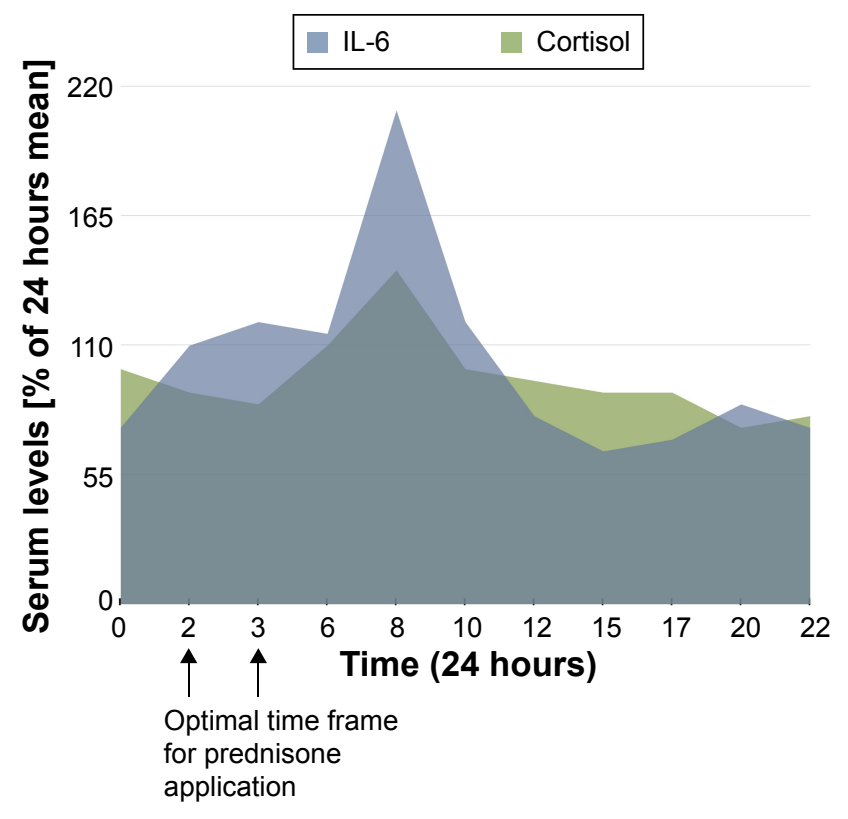

Figure I Chart showing the circadian rhythm of cortisol production as well as levels of IL-6 in RA patients.

Notes: Chart showing the circadian rhythm of cortisol production as well as levels of IL-6 in RA patients. The optimal time frame for prednisone application is indicated by the two arrows. Since this nocturnal medication is impractical, the formulation of MR prednisone with a time-delayed release of the acting agent was developed. Adapted with permission from (C) 2007 by the American College of Rheumatology. Straub RH, Cutolo M. Circadian rhythms in rheumatoid arthritis: implications for pathophysiology and therapeutic management. Arthritis Rheum. 2007;56(2):399-408. ${ }^{22}$

Abbreviations: IL-6, interleukin 6; MR, modified-release; RA, rheumatoid arthritis.

From the beginning of the $\mathrm{GC}$ use in clinical medicine, the negative impact of exogenous GCs on the HPA axis (including adrenal suppression) has been discussed. Historically, these effects were thought to be primarily related to dosage (suppression more likely at $>7.5 \mathrm{mg}$ prednisone equivalent per day) and therapy duration. Later investigations suggest that doses as small as $7.5 \mathrm{mg}$ prednisone equivalent are also capable of reducing the HPA axis response. ${ }^{26}$ An extensive review regarding this topic and the impact of modified-release (MR) prednisone has been drafted by Kirwan. ${ }^{27}$ There is evidence that an application of GCs in the morning is to be preferred since administration at midnight, for example, was shown to suppress endogenous cortisol production for 24 hours. GC use in the morning or afternoon, on the contrary, only caused a temporary suppression..$^{27,28}$ Further investigations indicated that multiple daily doses lead to a more pronounced HPA axis suppression, which, in turn, encouraged a single daily dose regimen. ${ }^{29,30}$ However, another study indicated that all dosing regimens interfere with the HPA axis. ${ }^{31}$ Taken together, the morning prescription of GCs around the endogenous peak is a widely accepted and established therapy regimen for decades now.

The recently introduced MR prednisone counteracts the HPA axis insufficiency without waking the patient and is a once-a-day application principle aiming to increase the GC levels before the endogenous peak in the morning.

\section{Pharmacology and drug development}

MR prednisone is a special tablet formulation of the common and clinically widely used GC prednisone.

Prednisone is an immunosuppressive drug in clinical use for more than half a century now. It is well known by physicians and commonly used, for example, for many rheumatic diseases, such as RA or giant cell arthritis, as well as pulmonary inflammatory conditions, such as bronchial asthma or chronic obstructive pulmonary disease. It has a half-life of approximately 2 hours and is usually administered in the morning for the reasons discussed earlier.

As a GC, prednisone is lipophilic and provides its effects basically on a genomic level utilizing the cytoplasmic glucocorticoid receptor (cGCR). After passing the plasma membrane, prednisone binds to the cGCR that is part of a multiprotein complex. The associated proteins dissociate and the remaining complex of GC-cGCR translocates into the nucleus and binds to specific DNA binding sites (glucocorticoid response elements) or interferes with the DNA by other transcription factors. ${ }^{32}$ This results in either increased production of antiinflammatory proteins by interaction with glucocorticoid response elements (transactivation) or a reduced expression of pro-inflammatory proteins due to negative interference with transcription factors (transrepression). For details, see Figure 2. Both processes contribute to the immunosuppressive effects, ${ }^{33}$ while the typical metabolic side effects are rather caused by transactivation. Since genes have to be activated and expressed, the genomic mode of action needs some time and is strictly dose-dependent with a ceiling effect at complete cGCR saturation (approximately 100-200 mg prednisone/day). In addition to the genomic effects, a nongenomic way of action is probably responsible for the clinically known fast effects, even if using high doses with all GCRs occupied. ${ }^{34}$ Details on this topic and the (non)genomic effects of prednisone are extensively discussed elsewhere. ${ }^{35}$

The main difference between MR prednisone and conventional prednisone is the time-delayed release of the active agent: taken orally at $10 \mathrm{pm}$, prednisone is released approximately 4 hours later (Figure 2), around $2 \mathrm{am}^{3}{ }^{36}$

MR prednisone was originally developed by Skye Pharma and Nitec Pharma (merged to Horizon Pharma in 2010) and got the brand name LODOTRA ${ }^{\circledR}$. It gained approval for general use in Europe in 2009 and is being commercialized and distributed by Mundipharma. In the USA, it has been 


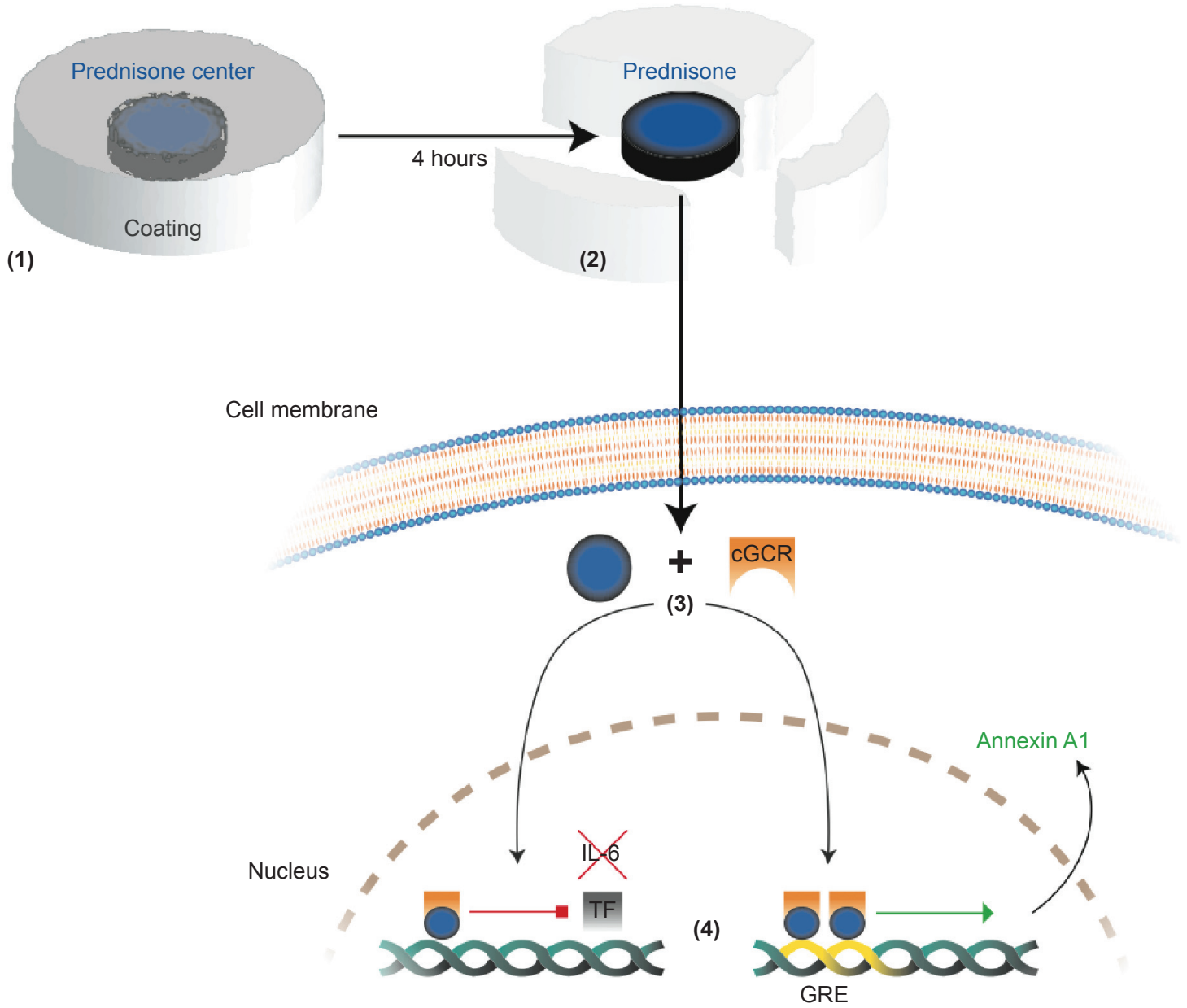

Figure 2 Simplified diagram showing ingestion, liberation, and way of action of modified-release prednisone.

Notes: Shown are ingestion, liberation, and way of action of modified-release prednisone in a simplified manner. The coating (I), displayed here in light gray, bursts approximately 4 hours after ingestion due to water penetration ${ }^{36}$ and releases the acting prednisone (blue) (2). As a lipophilic hormone, it is able to freely pass the plasma membrane. When reaching the cytosol, it binds (3) to the cytosolic glucocorticoid receptor (cGCR; orange), forming a complex and translocates into the nucleus. Within the nucleus, the complex either binds (4) to glucocorticoid response elements (GREs; yellow) as a dimer which in turn leads to increased production (green) of anti-inflammatory proteins, such as Annexin AI (right side), or interferes negatively with transcription factors (TF; gray), resulting in a suppression (red) of pro-inflammatory proteins, such as interleukin 6 (IL-6) (left side). The also proposed nongenomic effects are not shown for reasons of clarity and comprehensibility.

branded RAYOS ${ }^{\circledR}$ and is distributed by the licensee itself, Horizon Pharma, since 2012.

\section{Pharmacokinetics}

MR prednisone contains a prednisone center enveloped by a coat ensuring the release of prednisone approximately 4 hours after ingestion. It is designed to be taken at bedtime around $10 \mathrm{pm}$ in the evening with a prednisone release in the early morning, counteracting the cytokine peak. Highest plasma levels are reached approximately 6 hours after oral application. Pharmacological investigations show no substantial differences in pharmacokinetics besides the (wanted) delayed release of the GC from the tablet $\left(\mathrm{C}_{\max } 97 \%\right.$, $\left.\mathrm{AUC}_{0-\infty} 101 \%\right) .{ }^{37}$

It is important though to note that the application of MR prednisone in a fasted state results in a three- to fourfold reduction of its bioavailability; MR prednisone should consequently not be taken in fasted stated but after a light snack if the last meal has been more than 2-3 hours before. ${ }^{37}$

\section{Efficacy}

The efficacy of MR prednisone was the subject of investigation in two multicenter, randomized controlled trials (RCTs) named CAPRA (Circadian Administration of Prednisone in Rheumatoid Arthritis). While CAPRA-1 aimed to prove the efficacy and safety of MR prednisone compared to immediate-release (IR) prednisone, ${ }^{36}$ CAPRA-2 focused on MR prednisone as an additional GC therapy to an existing medication with disease-modifying antirheumatic drugs (DMARDs). ${ }^{38}$

CAPRA-1 originated in 2004 and included 288 patients already taking IR prednisone with an established 
RA diagnosis. ${ }^{8}$ Being randomly assigned $1: 1$ to get MR prednisone in the evening or continuing IR prednisone in the morning, the patients have been treated for 12 weeks in both arms. The trial was followed by a 9-month open-label extension (OLE) to evaluate the long-term efficacy and safety. Blinding in the original study was ensured using a doubledummy technique given twice a day. The results were most encouraging (Table 1): morning stiffness duration (primary endpoint) was significantly reduced when compared to the IR group $(-22.7 \%$ vs $-0.4 \%$; difference $=22.4 \%$ [ $95 \%$ confidence interval 0.49-44.30]; $P=0.045)$. The shown reduction went along with a significant decrease in IL-6 serum levels, supporting the hypothetical considerations of a prednisone chronotherapy. No difference between the two groups in any of the secondary endpoints (eg, $\triangle \mathrm{DAS} 28$, quality-of-lifemeasures, such as functional Health Assessment Questionnaire Disability Index) was seen.

Of greatest importance besides its efficacy though, the safety profile between MR and IR seems to be comparable with no difference in the rate of adverse events (AEs) (41\% each, Table 1). In both groups, the most common events have been worsening of RA, headache, abdominal pain, nasopharyngitis, and nausea at comparable frequencies.

The long-term OLE of CAPRA-1 showed similar results with reduced morning stiffness duration also for the patients being switched from IR to MR prednisone after the original 3 -month trial. ${ }^{39}$ The improvement was seen throughout the extension phase, first detected after 3 months of the openlabel treatment (Table 1). Consistent with the previous finding, the decrease of IL-6 serum levels was also detectable at the end of the OLE.

CAPRA-2 included 350 RA patients with an existing DMARD therapy and no IR prednisone medication 6 weeks prior to screening. ${ }^{38}$ All patients needed to have a morning stiffness duration of at least 45 minutes. The participants were double-blindly randomized 2:1 to either receive $5 \mathrm{mg}$ of MR prednisone or placebo in the evening. The primary endpoint was defined as the percentage of patients with a $20 \%$ improvement of RA signs and symptoms (ie, ACR20 response) at the end of study.

After 12 weeks, the MR prednisone group did not only show better response rates according to ACR20 (48\% vs $29 \%$, $P<0.001)$ but also to ACR50 ( $22 \%$ vs $10 \%, P<0.006)$. The superiority in ACR20 response was evident at week 2 already, sustaining the whole 12-week study duration $(P<0.005)$. Moreover, the reductions in morning stiffness $(-55 \%$ vs $-35 \% ; P<0.004)$ and fatigue as well as the improvements in physical function were found to be significantly greater in patients taking MR prednisone. Rates of AEs were comparable to CAPRA-1 with no significant differences between MR and placebo users (Tables 1 and 2).

Eventually, the results of the two CAPRA trials led to approval of MR prednisone in Europe and the USA.

Another important open-label RCT from Italy on MR prednisone investigated the impact of switching RA patients already using either IR prednisone or 6-methylprednisolone (6M-prednisolone) to a corresponding dose of MR prednisone.$^{40} \mathrm{~A}$ total of 950 patients (513 using IR prednisone and 437 using 6M-prednisolone) were included, switched on MR prednisone, and followed for 4 months. The investigators included 6M-prednisolone in their study since it is commonly used in Italy. After 16 weeks, morning stiffness - compared to baseline - decreased significantly from $58 \pm 37$ to $32 \pm 24$ minutes $(P<0.001)$ in all patients. Interestingly, the difference was more pronounced in the 6M-prednisolone group (6M-prednisolone: 67 vs 37 minutes, $P<0.001$; IR: 50 vs 27 minutes, $P<0.001$; $P$ for intergroup difference $<0.001$ ).

This result was somewhat surprising since 6M-prednisolone is known to have a higher inflammatory potential with resulting differing relative potencies compared to IR prednisone (parent drug: hydrocortisone $=1$, prednisone $=4$, $6 \mathrm{M}$-prednisolone $=5$ ). $\mathrm{GC}$ doses at baseline in both groups mirror these potencies (Table 1). The investigators therefore correctly expected a greater therapy difference in the IR prednisone group. Even if we cannot be sure of the reasons for this deviation, the authors provide a likely explanation when assigning the result to differences in compliance between 6M-prednisolone and IR formulations. ${ }^{40}$

In addition, the trial showed a significant reduction in disease activity (DAS28 score) in all patients $(4.2 \pm 1.4$ to $3.3 \pm 1.2 ; P<0.001)$ after switching to MR prednisone. There was no intergroup difference in this respect though.

The overall results of this open-label study strongly support the results of the CAPRA trials and ability of MR prednisone to counteract the insufficient production of endogenous cortisol in the morning, decreasing joint stiffness to a clinical meaningful extent.

Summing up the most important results of the reviewed trials, low-dose MR prednisone seems to be able to:

- reduce morning stiffness to a clinically meaningful extent (regardless of a prior GC therapy),

- decrease morning serum levels of IL-6, and

- induce a significantly higher amount of ACR20 and ACR50 responses in combination with DMARDs compared to DMARD monotherapy. 

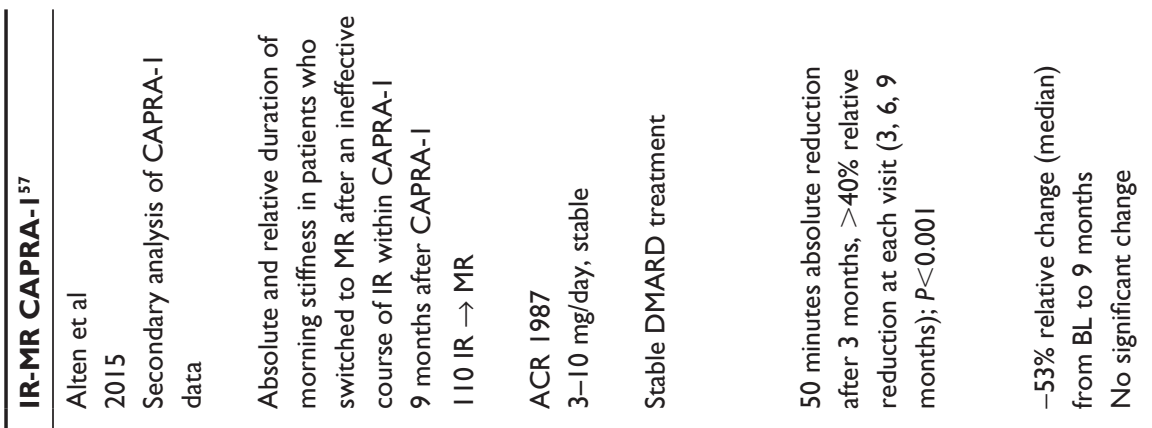

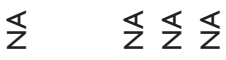
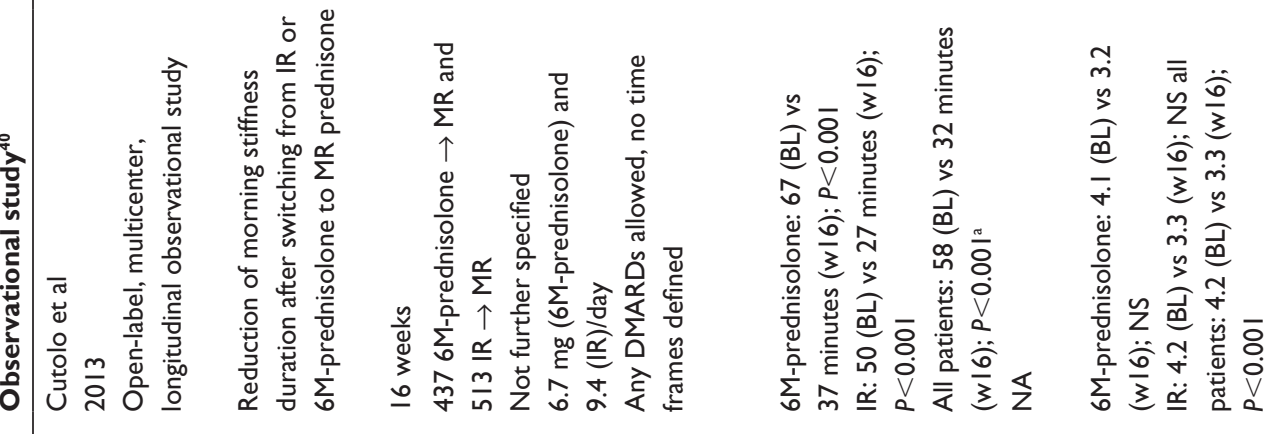

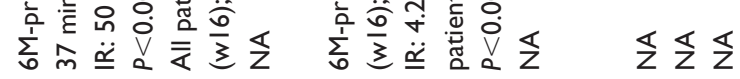
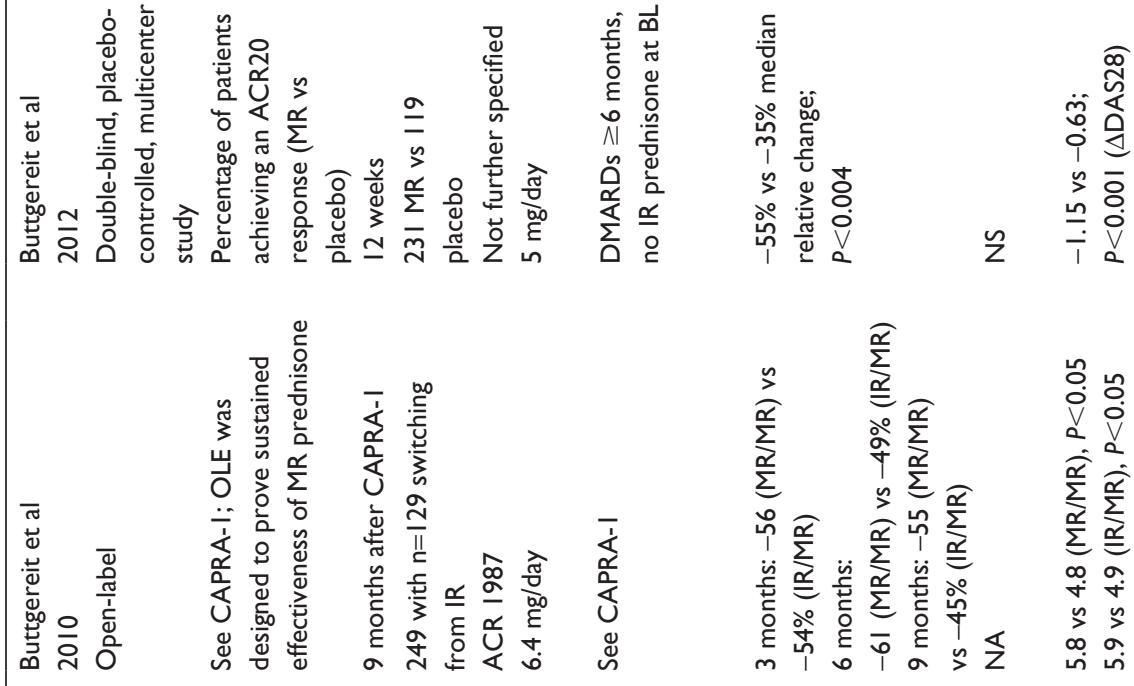

壬采采壬

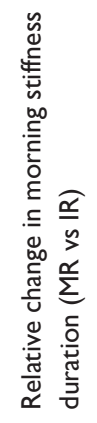

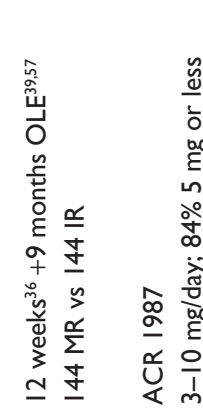

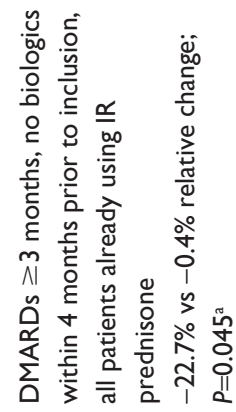

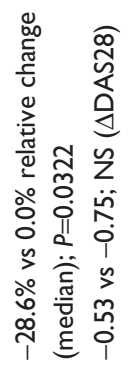

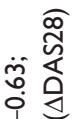
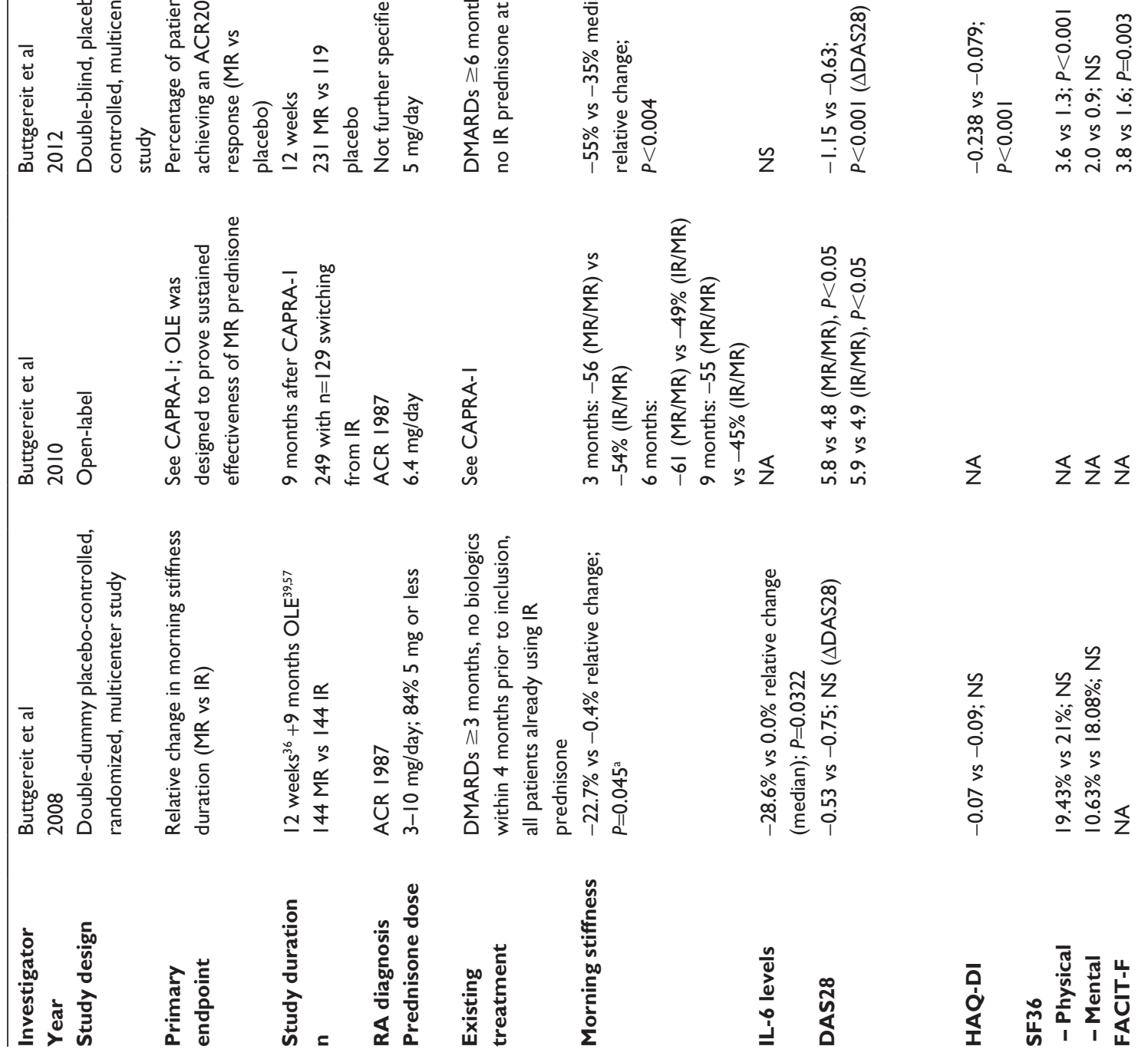
Due to the relatively short duration of the trials, the impact of MR prednisone on the progression of the disease itself could not be validated. When reviewing recent RCTs on the lowdose use of IR prednisone in RA, a DMARD-like potential of the drug could be hypothesized. In combination with synthetic DMARDs, such as methotrexate, radiographic joint damage is reduced when used over a longer period ( $2-4$ years), even after discontinuing IR prednisone. ${ }^{35}$ Since MR prednisone is virtually the same drug with almost identical pharmacodynamics and just an envelope ensuring a delayed liberation, a similar effect could be concluded on the long run.

The safety profile of MR prednisone will be discussed in detail in the following section of this paper.

\section{Safety and tolerability}

Since MR prednisone formally is a new drug, its efficacy has to be proven as well as its safety for the use in patients. Most safety data result from the registration trials mentioned in the "Efficacy" section. ${ }^{36,38,39}$

With prednisone being the actual acting agent, a hypothesis would be that side effects of MR prednisone should be comparable to IR formulations.

\section{Impact on the HPA axis}

No clinically evident AEs indicating an aggravated HPA axis suppression were observed in the CAPRA trials.

Furthermore, in order to examine possible effects of MR prednisone on the HPA axis, a small substudy "within" CAPRA-1 (Table 1) was undertaken. ${ }^{36,41}$ It consisted of measuring the cortisol plasma-levels after injection of synthetic corticotropin-releasing hormone $(\mathrm{CRH})$ in 28 patients at three different time points: baseline (prestudy IR treatment), 3 months (end of double-blind phase, IR or MR treatment), and 9 months (end of OLE, all patients on MR treatment). With no difference in mean increase of cortisol plasma concentrations after CRH injection at baseline compared to the end of OLE (5.5 \pm 4.37 vs $5.3 \pm 4.07 \mu \mathrm{g} / \mathrm{dL}$ ), no new HPA axis suppression occurred. Moreover, comparative testing between IR and MR during the double-blind period brought up no differences. Switching from IR to MR with beginning of the OLE phase also showed no difference in test results. The authors concluded that therapy with MR prednisone does not increase the risk of HPA axis insufficiency over 12 months of treatment. ${ }^{41}$

This argumentation can be followed, but the small sample size $(n=28)$ has to be considered. Another problem is the fact that at least one test per patient was done - meaning only some of the patients have been tested over the whole study duration of 12 months (including the OLE). In the review 
mentioned earlier, Kirwan reanalyzed the existing data from CAPRA-1 and extracted 17 patients who were tested at all of the three important time points. Interestingly, he found a significant increase of plasma cortisol after $\mathrm{CRH}$ administration under MR prednisone $(P<0.05)$, "showing that in this therapeutic study treatment over several months resulted in improved HPA axis responsiveness". ${ }^{27}$

Even with the sample size being small again, these results are encouraging and argue against a negative impact of MR prednisone upon the HPA axis. In fact, quite the opposite could be the case.

\section{Side effects in general}

In general, the incidence of AEs was low in both trials with MR prednisone showing a slightly lower (placebo, not significant) or comparable (IR prednisone) incidence (Table 1). AEs considered being treatment-related were also comparable throughout both studies. The most common AEs seen were arthralgia, RA flares, abdominal pain, nasopharyngitis, headache, and flush (Table 2). All cases of flush could be attributed to the CRH tests discussed in detail earlier.

Since MR prednisone was given in doses usually considered being low ( $\leq 5-10 \mathrm{mg} /$ day), these findings are perfectly in line with safety data reported from trials on the low-dose use of IR prednisone in RA. Both an excellent review on this topic and a meta-analysis show a modest risk for low-dose prednisone in RA patients. ${ }^{42,43}$ These conclusions have been confirmed in recent RCTs. ${ }^{44-46}$ It is therefore considered to be safe to use low-dose IR prednisone in RA when thoroughly monitored by the attending rheumatologist. ${ }^{35}$

Hypothesizing that side effects of MR prednisone are comparable, this consideration can be applied on this new formulation as well. It is further supported by the findings of CAPRA-1 and -2 as shown in Table 2.

Nevertheless, it clearly is important to have the metabolic side effects of GCs, such as hypertension, impaired glucose tolerance, and weight gain, in mind. Available safety data derives from relatively short trials mentioned above. The long-term impact of both the low-dose use of IR as well as MR prednisone on a patient's health is therefore much less clear and should be the subject of further clinical trials.

The long-term impact of both the low-dose use of IR as well as MR prednisone on a patient's health is therefore much less clear and should be the subject of further clinical trials.

\section{Quality of life}

RA has a negative impact on the physical function and everyday quality of life. Besides, it often causes burdensome constitutional unspecific symptoms, such as fatigue.
Table 2 AEs reported in the clinical trials comparing MR prednisone with IR prednisone and placebo, respectively ${ }^{36,38,39}$

\begin{tabular}{|c|c|c|c|}
\hline $\mathrm{AE}$ & MR (\%) & IR (\%)* & Placebo (\%) \\
\hline Arthralgia & $1 *-10.4^{\ddagger}$ & 2 & 20.2 \\
\hline RA flare & $6.5^{\ddagger}-8^{*}$ & 9 & 9.2 \\
\hline Abdominal pain & $4^{*}$ & 6 & - \\
\hline Nasopharyngitis & $3^{*}-4.8^{\ddagger}$ & 6 & 3.4 \\
\hline Headache & $3.9 \neq-4 *$ & 3 & 4.2 \\
\hline Flush & $3^{*}$ & 4 & - \\
\hline Weight increase & $2.4^{\#}$ & - & - \\
\hline Hypertension & $2^{*}-2.2^{\ddagger}$ & 2 & 0.8 \\
\hline Chest pain & $2^{*}$ & 2 & - \\
\hline Nausea/vomiting & $1.3^{\ddagger}-4^{*}$ & 3 & 0.8 \\
\hline Diarrhea & $1.7^{\ddagger}$ & - & 0.8 \\
\hline Rash & $1.7^{\ddagger}$ & - & 0.8 \\
\hline Gastritis & $1.6^{\#}$ & - & - \\
\hline Back pain & $1.3^{\ddagger}$ & - & 0.8 \\
\hline Bronchitis & $1 *-1.3^{\ddagger}$ & 4 & 4.2 \\
\hline Vertigo & I* & 3 & - \\
\hline Dyspepsia & $I^{*}$ & 2 & - \\
\hline $\begin{array}{l}\text { Upper respiratory } \\
\text { tract infection }\end{array}$ & $I^{*}$ & 2 & - \\
\hline Peripheral edema & $0.9^{\ddagger}$ & - & 1.7 \\
\hline Hematuria & $0.4^{\ddagger}$ & - & 2.5 \\
\hline
\end{tabular}

Notes: All values represent percentage of patients in the distinct group experiencing the named AEs. Missing values indicate that the named AE did not occur. The according clinical trial is marked as follows: *, CAPRA-I; ‡, CAPRA-2; ", CAPRA-I OLE.

Abbreviations: AE, adverse event; CAPRA, Circadian Administration of Prednisone in Rheumatoid Arthritis; IR, immediate-release prednisone; MR, modified-release prednisone; OLE, open-label extension; RA, rheumatoid arthritis.

The CAPRA-2 study compared patients receiving a new MR prednisone therapy against placebo showing that the additional treatment has a positive impact on morning stiffness and improves response rates. ${ }^{38}$ Besides the primary outcomes, the investigators of CAPRA-2 also studied the influence on fatigue and quality of life-related measurements. During the 12-week study, the RA-evaluated Functional Assessment of Chronic Illness Therapy-Fatigue score improved significantly in the MR prednisone group (least squares mean difference 2.2, $P=0.0032$ ) compared to placebo. ${ }^{47}$ This improvement in Functional Assessment of Chronic Illness Therapy-Fatigue was associated with ACR20 responsiveness in both groups. Furthermore, other quality of life markers, such as the 36-item Short-Form health survey or the Health Assessment Questionnaire Disability Index, also showed a significant improvement in the MR prednisone group ( $P=0.001$ and $<0.001$, respectively). ${ }^{38,47}$

These findings indicate a benefit with regard to patientreported outcomes for MR prednisone as an addition to an existing DMARD regime in prednisone-naïve patients. Reviewing the literature, there is some evidence that IL-6 levels might be associated with fatigue in inflammatory conditions in general and RA in particular. ${ }^{48-50}$ Based on the 
causative connection between IL-6 and morning stiffness, the fatigue improvement found in CAPRA-2 seems to be conclusive. It is also well in line with the reduction of IL-6 levels maintained for 12 months under MR prednisone seen in CAPRA- $1 .{ }^{39}$

Interestingly, CAPRA-1 did not show any significant improvements in such quality of life measurements (Health Assessment Questionnaire Disability Index and 36-item Short-Form; Functional Assessment of Chronic Illness Therapy-Fatigue not available, Table 1) for MR prednisone users. ${ }^{36}$ This first clinical trial on MR prednisone was designed to prove superiority compared to IR prednisone in reducing morning stiffness though. The missing positive impact on markers of quality of life cannot be readily explained: improved morning function is thought to influence the patient's mood as well as his or her participation on everyday (working) life positively.

Overall, the two studies are difficult to compare since the patient population as well as the inclusion criteria were different. Furthermore, the primary and secondary outcome parameters varied between the studies. Further studies are necessary to clearly define the effect of MR prednisone on these patient-reported outcomes.

Both CAPRA trials showed that MR prednisone is a promising opportunity for both existing and new prednisone users. With morning stiffness being reduced at least in duration, the potential gain for the individual RA patient is encouraging. Additionally, there might also be a positive impact on fatigue.

\section{Cost effectiveness}

MR prednisone is a rather new drug with a patent held by Horizon Pharma. It has a promising potential to reduce morning stiffness, one of the most important clinical symptoms of RA.

With a prevalence of approximately $1 \%$, RA is a common disease with high societal impact: total costs are estimated to be $\$ 19.3$ billion annually in the USA.${ }^{51}$ Direct per-patient costs were calculated to be $\$ 4,677$ in the USA (2008) and $€ 4,737$ in Germany (2002). ${ }^{52}$ A current study shows an increase of direct costs up to $€ 8,206$ (patients $<65$ years) and $€ 6,221$ (patients $\geq 65$ years) in 2011 in Germany. ${ }^{52}$ This increase is mainly caused by the use of biologic agents whose costs have reached a plateau with no further increase to be expected. ${ }^{52}$

To date, 30 pills of Lodotra ${ }^{\circledR}$ cost approximately $€ 37$ in Germany, which is comparable to the UK with a cost of approximately $£ 27 .{ }^{53}$ Cost per pill is therefore approximately $€ 1$ or $£ 1$. Compared to common prednisone tablets, this is more than 20 times as much with yearly costs of $£ 300-600$, depending on the individual dose. A mathematical modelbased analysis for the UK suggests a cost effectiveness for the usage of MR prednisone instead of IR prednisone for at least 1 year of treatment within the National Health Services environment. ${ }^{53}$ Furthermore, calculations resting on the data gained from CAPRA-2 suggest that MR prednisone is able to postpone the beginning of an expensive biologic therapy and therefore saves costs. ${ }^{54}$ In the latter study, indications for initiating a biologic therapy from the Netherlands, Belgium, and the UK have been considered.

The calculations mentioned earlier are all based on prices in Europe. In the USA, however, the same amount of Rayos ${ }^{\circledR}$ (30 pills) is sold for over $\$ 1,600$ (cost per-pill: $\$ 50) .{ }^{55}$ With IR prednisone being priced $13 \notin$ per tablet, MR prednisone is more than 350-fold as expensive as the conventional product. Annual costs for MR prednisone only would be as much as approximately $\$ 20,000$ (by comparison: etanercept $\$ 14,385$ / year, infliximab $\$ 19,283 /$ year). ${ }^{56}$

This enormous difference between prices in the USA and Europe raises the question whether the use of MR prednisone can be justified by its actual impact and relevance for the individual patient. Just recently, this question has been addressed by a colleague from the USA. ${ }^{55}$

Economical thoughts on drugs or medical products have to be accompanied by ethical considerations in respect of the individual patient as well as public interest (particularly in context of statutory health care systems). Expensive treatment options can be very valuable in the individual while totally useless in others. The clinical trials leading to the approval of MR prednisone showed that the drug is able to improve morning stiffness to a clinically meaningful extent. Morning stiffness in RA patients impairs everyday life as well as the ability to participate on working life without restrictions. With MR prednisone potentially counteracting the impact of morning stiffness at least partly, not only the patient's private life can be improved due to reducing the symptoms but also the negative (financial) consequences of impaired working capacity, sick days, or even early retirement for the whole society are addressed. Therefore, an individual attempt in patients with pronounced morning stiffness seems mandatory for the rationally acting doctor - and also from an economical point of view.

\section{Conclusion}

GCs have been used in clinical medicine, in particular in inflammatory conditions, such as RA, for more than half a century now. Among GCs, prednisone is sort of a lead substance and the most commonly used substitute of its class of 
drugs. The potency of all other substitutes is usually declared relative to prednisone ("prednisone equivalent").

The probably most recent and clinically important invention (besides selective GC receptor agonists) is MR prednisone targeting the nocturnal cytokine peak and therefore reducing morning stiffness duration in RA successfully. Morning stiffness is not only one of the most important challenges in treating the disease but also an impairing symptom with particular relevance for the patient. Halls et $\mathrm{al}^{13}$ framed it to the point: "(Morning) stiffness is more than just duration and severity". Nevertheless, reducing its duration contributes to the patient's well-being and possibly improves the impairments in everyday life. On the long run, MR prednisone could also help in retarding joint destruction due to its pharmacodynamics being most likely identical to conventional IR prednisone. For the latter one, recent clinical trials were able to show disease-modifying potential when used in low daily doses as an addition to DMARDs, such as methotrexate.

In particular, in patients with pronounced morning stiffness, the use of MR prednisone should be considered by all means. In this regard, the stiffness reduction was proven for both new and existing GC users. The comprehensible question of cost effectiveness of this therapy, however, is difficult to answer. Severity of symptoms, individually expected impacts on the disease, and especially costs of prescription have to be taken into account by the attending rheumatologist. While cost effectiveness for Europe could be calculated, current drug costs in the USA make such a conclusion less easy.

The existing data on MR prednisone suggest a superior efficacy in reducing RA-related morning stiffness while bearing a safety profile comparable to conventional prednisone formulations. This also includes a possible impact on the HPA axis. To prove its long-term safety and efficacy, more clinical trials are needed in order to strengthen the gained results. Nevertheless, the importance of MR prednisone in clinical routine will grow further in distinct patient populations.

\section{Author contributions}

$\mathrm{MK}$ and $\mathrm{CB}$ both reviewed the literature, drafted the manuscript, approved the final version to be published and agree to be accountable for all aspects of the work.

\section{Disclosure}

$\mathrm{CB}$ has received lecture fees from Merck KGaA, MSD, Mundipharma, and Pfizer. MK declares no financial conflict of interest.

\section{References}

1. Myasoedova E, Crowson CS, Kremers HM, Therneau TM, Gabriel SE. Is the incidence of rheumatoid arthritis rising? Results from Olmsted County, Minnesota, 1955-2007. Arthritis Rheum. 2010;62(6):1576-1582.

2. Crowson CS, Matteson EL, Myasoedova E, et al. The lifetime risk of adult-onset rheumatoid arthritis and other inflammatory autoimmune rheumatic diseases. Arthritis Rheum. 2011;63(3):633-639.

3. Scott DL, Wolfe F, Huizinga TW. Rheumatoid arthritis. Lancet. 2010; 376(9746):1094-1108.

4. van der Kooij SM, de Vries-Bouwstra JK, Goekoop-Ruiterman YP, et al. Patient-reported outcomes in a randomized trial comparing four different treatment strategies in recent-onset rheumatoid arthritis. Arthritis Rheum. 2009;61(1):4-12.

5. Voskuyl AE. The heart and cardiovascular manifestations in rheumatoid arthritis. Rheumatology. 2006;45 Suppl 4:iv4-iv7.

6. Vliet Vlieland TP, Zwinderman AH, Breedveld FC, Hazes JM. Measurement of morning stiffness in rheumatoid arthritis clinical trials. J Clin Epidemiol. 1997;50(7):757-763.

7. Phillips S, Dow L. Impact of impaired morning function on quality of life in rheumatoid arthritis: results of an exploratory patient survey. Int J Clin Rheumatol. 2012;7(6):597-606.

8. Arnett FC, Edworthy SM, Bloch DA, et al. The American Rheumatism Association 1987 revised criteria for the classification of rheumatoid arthritis. Arthritis Rheum. 1988;31(3):315-324.

9. Aletaha D, Neogi T, Silman AJ, et al. 2010 Rheumatoid arthritis classification criteria: an American College of Rheumatology/European League Against Rheumatism collaborative initiative. Arthritis Rheum. 2010;62(9):2569-2581.

10. Kaarela K, Kauppi J, Kauppi M. The 2010 ACR/EULAR classification criteria for rheumatoid arthritis in the Heinola inception cohort diagnoses confirmed by long-term follow-up. Clin Rheumatol. 2012; 31(3):547-551.

11. Boers M, Buttgereit F, Saag K, et al. What is the relationship between morning symptoms and measures of disease activity in patients with rheumatoid arthritis? Arthritis Care Res. Epub 2015 Mar 25.

12. Tuominen R, Tuominen S, Mottonen T. How much is a reduction in morning stiffness worth to patients with rheumatoid arthritis? Scand J Rheumatol Suppl. 2011;125:12-16.

13. Halls S, Dures E, Kirwan J, et al. Stiffness is more than just duration and severity: a qualitative exploration in people with rheumatoid arthritis. Rheumatology. 2015;54(4):615-622.

14. Meusch U, Rossol M, Baerwald C, Hauschildt S, Wagner U. Outside-toinside signaling through transmembrane tumor necrosis factor reverses pathologic interleukin-1beta production and deficient apoptosis of rheumatoid arthritis monocytes. Arthritis Rheum. 2009;60(9):2612-2621.

15. Rossol M, Kraus S, Pierer M, Baerwald C, Wagner U. The CD14(bright) CD16+ monocyte subset is expanded in rheumatoid arthritis and promotes expansion of the Th17 cell population. Arthritis Rheum. 2012; 64(3):671-677.

16. Krasselt M, Baerwald C, Wagner U, Rossol M. CD56+ monocytes have a dysregulated cytokine response to lipopolysaccharide and accumulate in rheumatoid arthritis and immunosenescence. Arthritis Res Ther. 2013;15(5):R139.

17. Meusch U, Klingner M, Baerwald C, Rossol M, Wagner U. Deficient spontaneous in vitro apoptosis and increased tmTNF reverse signalinginduced apoptosis of monocytes predict suboptimal therapeutic response of rheumatoid arthritis to TNF inhibition. Arthritis Res Ther. 2013;15(6):R219.

18. Meusch U, Krasselt M, Rossol M, Baerwald C, Klingner M, Wagner U. In vitro response pattern of monocytes after tmTNF reverse signaling predicts response to anti-TNF therapy in rheumatoid arthritis. $J$ Transl Med. 2015;13:256.

19. Arvidson NG, Gudbjornsson B, Elfman L, Ryden AC, Totterman TH, Hallgren R. Circadian rhythm of serum interleukin-6 in rheumatoid arthritis. Ann Rheum Dis. 1994;53(8):521-524.

20. Cutolo M, Villaggio B, Otsa K, Aakre O, Sulli A, Seriolo B. Altered circadian rhythms in rheumatoid arthritis patients play a role in the disease's symptoms. Autoimmun Rev. 2005;4(8):497-502. 
21. Khurana R, Berney SM. Clinical aspects of rheumatoid arthritis. Pathophysiology. 2005;12(3):153-165.

22. Straub RH, Cutolo M. Circadian rhythms in rheumatoid arthritis: implications for pathophysiology and therapeutic management. Arthritis Rheum. 2007;56(2):399-408.

23. Arvidson NG, Gudbjornsson B, Larsson A, Hallgren R. The timing of glucocorticoid administration in rheumatoid arthritis. Ann Rheum Dis. 1997;56(1):27-31.

24. Spies CM, Straub RH, Cutolo M, Buttgereit F. Circadian rhythms in rheumatology - a glucocorticoid perspective. Arthritis Res Ther. 2014; 16 Suppl 2:S3

25. Straub RH, Paimela L, Peltomaa R, Scholmerich J, Leirisalo-Repo M. Inadequately low serum levels of steroid hormones in relation to interleukin-6 and tumor necrosis factor in untreated patients with early rheumatoid arthritis and reactive arthritis. Arthritis Rheum. 2002;46(3):654-662.

26. Kirwan JR, Hickey SH, Hallgren R, et al. The effect of therapeutic glucocorticoids on the adrenal response in a randomized controlled trial in patients with rheumatoid arthritis. Arthritis Rheum. 2006;54(5): 1415-1421.

27. Kirwan JR. Targeting the time of day for glucocorticoid delivery in rheumatoid arthritis. Int J Clin Rheum. 2011;6(3):273-279.

28. Nichols T, Nugent CA, Tyler FH. Diurnal variation in suppression of adrenal function by glucocorticoids. J Clin Endocrinol Metab. 1965;25: 343-349.

29. Klinefelter HF, Winkenwerder WL, Bledsoe T. Single daily dose prednisone therapy. JAMA. 1979;241(25):2721-2723.

30. Grant SD, Forsham PH, DiRaimondo VC. Suppression of 17hydroxycorticosteroids in plasma and urine by single and divided doses of triamcinolone. N Engl J Med. 1965;273(21):1115-1118.

31. Jasani MK, Diver MJ, Bell AM, Dalakos TG, Buchanan WW. Some clinical observations on the diurnal rhythm of plasma cortisol (11-OHCS) in patients with rheumatoid arthritis treated with oral corticosteroid drugs. Curr Med Res Opin. 1974;2(7):373-386.

32. Almawi WY, Melemedjian OK. Molecular mechanisms of glucocorticoid antiproliferative effects: antagonism of transcription factor activity by glucocorticoid receptor. J Leukoc Biol. 2002;71(1):9-15.

33. Coutinho AE, Chapman KE. The anti-inflammatory and immunosuppressive effects of glucocorticoids, recent developments and mechanistic insights. Mol Cell Endocrinol. 2011;335(1):2-13.

34. Buttgereit F, Scheffold A. Rapid glucocorticoid effects on immune cells. Steroids. 2002;67(6):529-534

35. Krasselt M, Baerwald C. The current relevance and use of prednisone in rheumatoid arthritis. Expert Rev Clin Immunol. 2014;10(5):557-571.

36. Buttgereit F, Doering G, Schaeffler A, et al. Efficacy of modified-release versus standard prednisone to reduce duration of morning stiffness of the joints in rheumatoid arthritis (CAPRA-1): a double-blind, randomised controlled trial. Lancet. 2008;371(9608):205-214.

37. Derendorf H, Ruebsamen K, Clarke L, Schaeffler A, Kirwan JR. Pharmacokinetics of modified-release prednisone tablets in healthy subjects and patients with rheumatoid arthritis. J Clin Pharmacol. 2013; 53(3):326-333.

38. Buttgereit F, Mehta D, Kirwan J, et al. Low-dose prednisone chronotherapy for rheumatoid arthritis: a randomised clinical trial (CAPRA-2). Ann Rheum Dis. 2013;72(2):204-210.

39. Buttgereit F, Doering G, Schaeffler A, et al. Targeting pathophysiological rhythms: prednisone chronotherapy shows sustained efficacy in rheumatoid arthritis. Ann Rheum Dis. 2010;69(7):1275-1280.

40. Cutolo M, Iaccarino L, Doria A, Govoni M, Sulli A, Marcassa C. Efficacy of the switch to modified-release prednisone in rheumatoid arthritis patients treated with standard glucocorticoids. Clin Exp Rheumatol. 2013; 31(4):498-505.
41. Alten R, Doring G, Cutolo M, et al. Hypothalamus-pituitary-adrenal axis function in patients with rheumatoid arthritis treated with nighttimerelease prednisone. $J$ Rheumatol. 2010;37(10):2025-2031.

42. Hoes JN, Jacobs JW, Verstappen SM, Bijlsma JW, Van der Heijden GJ. Adverse events of low- to medium-dose oral glucocorticoids in inflammatory diseases: a meta-analysis. Ann Rheum Dis. 2009;68(12): 1833-1838.

43. Da Silva JA, Jacobs JW, Kirwan JR, et al. Safety of low dose glucocorticoid treatment in rheumatoid arthritis: published evidence and prospective trial data. Ann Rheum Dis. 2006;65(3):285-293.

44. Malysheva OA, Wahle M, Wagner U, et al. Low-dose prednisolone in rheumatoid arthritis: adverse effects of various disease modifying antirheumatic drugs. $J$ Rheumatol. 2008;35(6):979-985.

45. Bakker MF, Jacobs JW, Welsing PM, et al. Low-dose prednisone inclusion in a methotrexate-based, tight control strategy for early rheumatoid arthritis: a randomized trial. Ann Intern Med. 2012;156(5):329-339.

46. Montecucco C, Todoerti M, Sakellariou G, Scire CA, Caporali R. Lowdose oral prednisone improves clinical and ultrasonographic remission rates in early rheumatoid arthritis: results of a 12-month open-label randomised study. Arthritis Res Ther. 2012;14(3):R112.

47. Alten R, Grahn A, Holt RJ, Rice P, Buttgereit F. Delayed-release prednisone improves fatigue and health-related quality of life: findings from the CAPRA-2 double-blind randomised study in rheumatoid arthritis. RMD Open. 2015;1(1):e00134.

48. Rief W, Mills PJ, Ancoli-Israel S, Ziegler MG, Pung MA, Dimsdale JE. Overnight changes of immune parameters and catecholamines are associated with mood and stress. Psychosom Med. 2010;72(8):755-762.

49. Rohleder N, Aringer M, Boentert M. Role of interleukin-6 in stress, sleep, and fatigue. Ann N Y Acad Sci. 2012;1261:88-96.

50. Fragiadaki K, Tektonidou MG, Konsta M, Chrousos GP, Sfikakis PP. Sleep disturbances and interleukin 6 receptor inhibition in rheumatoid arthritis. J Rheumatol. 2012;39(1):60-62.

51. Birnbaum H, Pike C, Kaufman R, Marynchenko M, Kidolezi Y, Cifaldi M. Societal cost of rheumatoid arthritis patients in the US. Curr Med Res Opin. 2010;26(1):77-90.

52. Huscher D, Mittendorf T, von Hinuber U, et al. Evolution of cost structures in rheumatoid arthritis over the past decade. Ann Rheum Dis. 2015; 74(4):738-745.

53. Dunlop W, Iqbal I, Khan I, Ouwens M, Heron L. Cost-effectiveness of modified-release prednisone in the treatment of moderate to severe rheumatoid arthritis with morning stiffness based on directly elicited public preference values. Clinicoecon Outcomes Res. 2013;5:555-564.

54. Boers M, Buttgereit F. A simple model that suggests possible cost savings when modified-release prednisone $5 \mathrm{mg}$ /day is added to current treatment in patients with active rheumatoid arthritis. Rheumatology. 2013;52(8):1435-1437.

55. Conn DL. Is the availability of delayed release prednisone an important clinical advance? Arthritis Care Res. Epub 2015 Sep 28.

56. Curtis JR, Chastek B, Becker L, et al. Cost and effectiveness of biologics for rheumatoid arthritis in a commercially insured population. J Manag Care Spec Pharm. 2015;21(4):318-329.

57. Alten R, Holt R, Grahn A, et al. Morning stiffness response with delayed-release prednisone after ineffective course of immediate-release prednisone. Scand J Rheumatol. 2015;44(5):354-358.

58. Cutolo M, Straub RH, Buttgereit F. Circadian rhythms of nocturnal hormones in rheumatoid arthritis: translation from bench to bedside. Ann Rheum Dis. 2008;67(7):905-908. 


\section{Publish your work in this journal}

Drug Design, Development and Therapy is an international, peerreviewed open-access journal that spans the spectrum of drug design and development through to clinical applications. Clinical outcomes, patient safety, and programs for the development and effective, safe, and sustained use of medicines are a feature of the journal, which

has also been accepted for indexing on PubMed Central. The manuscript management system is completely online and includes a very quick and fair peer-review system, which is all easy to use. Visit http://www.dovepress.com/testimonials.php to read real quotes from published authors.

Submit your manuscript here: http://www.dovepress.com/drug-design-development-and-therapy-journal 